|| ISSN(online): 2589-8698 || ISSN(print): 2589-868X || International Journal of Medical and Biomedical Studies

Available Online at www.ijmbs.info

PubMed (National Library of Medicine ID: 101738825)

Index Copernicus Value 2018: 75.71

Volume 3, Issue 4; April: 2019; Page No. 150-153

\title{
CORRELATION OF SERUM VITAMIN D LEVEL WITH MORTALITY IN PATIENTS WITH SEPSIS
}

\author{
Pramod Sood ${ }^{1}$, Manpreet Kaur ${ }^{2}$ \\ ${ }^{1}$ Associate Professor, Department of Critical Care Medicine, Dayanand Medical College and Hospital \\ Ludhiana \\ ${ }^{2}$ Reader, Department of oral pathology, Pacific dental College Udaipur.
}

Article Info: Received 24 March 2019; Accepted 20 April. 2019

Cite this article as: Sood, P., \& Kaur, M. (2019). CORRELATION OF SERUM VITAMIN D LEVEL WITH MORTALITY IN PATIENTS WITH SEPSIS. International Journal of Medical and Biomedical Studies, 3(4).

DOI: https://doi.org/10.32553/ijmbs.v3i4.206

Address for Correspondence: Pramod Sood, Associate Professor, Department of Critical Care Medicine, Dayanand Medical College and Hospital Ludhiana

Conflict of interest: No conflict of interest.

\section{Abstract}

Background: Vitamin D insufficiency is a common global phenomenon. Its insufficiency is also reported to be common in hospitalized patients and particularly in critically ill patients.

Methods: Following ethical approval, consent will be sought from either the patient or assent from a near relative. Successive patients admitted to the medical emergency and ICU at tertiary care health center who fulfill the following criteria for sepsis, within a $24 \mathrm{~h}$ time window, were included in the study.

Results: A total of 50 patients were enrolled in the study, 37 (74.00\%) were discharged whereas $13(26.00 \%)$ patients expired. The majority of patients, irrespective of outcome had Vitamin D deficiency. Although normal Vitamin D levels were observed in relatively higher proportion of patients who were discharged ( 29 patients) as compared to those who expired (12 patients) yet this difference was not significant statistically $(P=0.481)$.

Conclusion: Vitamin D deficiency leads to increased risk of mortality in the critically ill patients.

Keywords: Sepsis, Vitamin D deficiency,Mortality.

\section{Introduction}

Although vitamin D (vit D) is classified as a fatsoluble vitamin that plays an important role in bone metabolism, it is also a steroid hormone with pleiotropic effects. With its pleiotropic effects, vit $D$ regulates immunity, inflammation, cell proliferation, differentiation, apoptosis, and angiogenesis. Several recent studies demonstrate a close relationship between vit $D$ insufficiency and various systemic disorders associated with significant morbidity and mortality. ${ }^{1,2}$
Vitamin D insufficiency is a common global phenomenon. Its insufficiency is also reported to be common in hospitalized patients and particularly in critically ill patients ${ }^{3}$. The incidence of vit $D$ insufficiency in critically ill patients has been reported to range from $26 \%$ to $82 \%{ }^{4}$. This insufficiency may worsen existing immune and metabolic dysfunctions in critically ill patients, leading to worse outcomes ${ }^{5}$.

The purpose of this study was to evaluate vit D status in our critically ill patients and to demonstrate the relationship between vit $D$ status and mortality. 


\section{Material and Methods}

This was a case-control study. All patients over 18 years of age who were referred to the emergency department due to sepsis, were assessed for enrollment in the study as the case group $(n=50)$.

Inclusion criteria: all adult patients admitted in Medical Emergency or in Intensive Care Unit (ICU) with features of sepsis, severe sepsis, or septic shock were enrolled.

Exclusion criteria: pregnant and lactating mothers, postcardiopulmonary resuscitated patient, not giving consent, taking multivitamin, or food supplements which contain Vitamin D, malabsorption syndromes or patients with chronic diarrhea. Informed consent was taken from every patient before being included in the study. The study was approved by Local Ethical Committee.

Patients were evaluated on the basis of history, examination (general and systemic), and appropriate investigations were done (hemoglobin, total leukocyte count, differential leukocyte count, platelet count, hematocrit, fasting lipid profile, random blood sugar, $\mathrm{pH}$, $\mathrm{Na}+, \mathrm{K}+$, ionic calcium, magnesium, phosphate, bicarbonate, serum urea, serum creatinine, glycated hemoglobin, fasting and postprandial blood sugar, liver function tests - serum bilirubin, serum glutamic oxaloacetic transaminases, serum glutamic pyruvic transaminases, serum protein, serum albumin, Creactive protein (CRP), thyroid-stimulating hormone, T3, T4, serum lipase).

Additional tests were also done when needed like electrocardiography, to search for the source of infection (urine routine and microscopy and culture sensitivity, blood culture and sensitivity, sputum culture and sensitivity, X-ray chest). After initial patient evaluation and investigations, the patients were graded according to Glasgow coma scale (GCS) and Acute Physiology and Chronic Health Evaluation (APACHE) II scoring systems.

\section{Statistical Analysis}

To compare quantitative variables between the two groups, if the data were normally distributed, an independent-samples t-test was used. To compare quantitative variables between more than two groups, one-way ANOVA was used to analyze the data. If the data were not normally distributed, equivalent nonparametric tests were used. All data were analyzed with SPSS 16.0 statistical software (SPSS Inc., Champaign, IL, USA).

We used the Pearson chi-squared test to analyze qualitative variables, and if the data were not eligible for the test, other appropriate tests were used, such as Fisher's exact or the likelihood ratio. To assess correlations between quantitative data, the Pearson correlation test was used. In all cases, a significance level of P < 0.05 was set.

Results:

\section{Table 1: Distribution of cases according to outcome}

\begin{tabular}{|l|l|l|}
\hline Outcome & Number of case & Percentage \\
\hline Discharge & 37 & 74 \\
\hline Dead & 13 & 26 \\
\hline
\end{tabular}

A total of 50 patients were enrolled in the study, 37 (74.00\%) were discharged whereas $13(26.00 \%)$ patients expired.

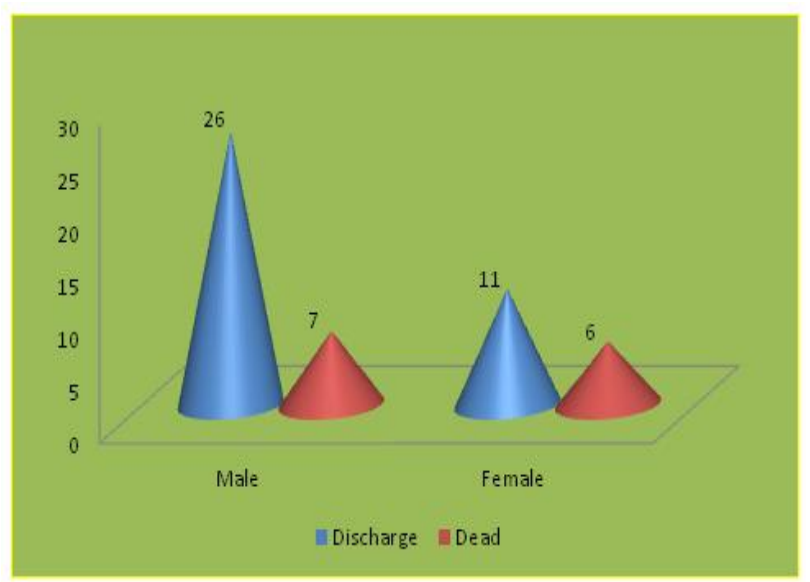

Out of total 50 patients, $74.00 \%$ patients were male and $26.00 \%$ patients were male. Statistically, there was no significant association between gender and outcome. 
Table 2: Association between Vitamin D levels and outcome

\begin{tabular}{|l|l|l|l|}
\hline Outcome & Discharge & Dead & Total \\
\hline Deficiency & 29 & 12 & 41 \\
\hline Normal & 8 & 1 & 9 \\
\hline Total & 37 & 13 & 50 \\
\hline$p$-value & 0.481 & \\
\hline
\end{tabular}

A total of 50 patients were enrolled in the study, 37 (74.00\%) were discharged whereas $13(26.00 \%)$ patients expired. The majority of patients, irrespective of outcome had Vitamin D deficiency. Although normal Vitamin D levels were observed in relatively higher proportion of patients who were discharged (29 patients) as compared to those who expired (12 patients) yet this difference was not significant statistically $(P=0.481)$.

\section{Discussion}

A total of 50 patients were enrolled in the study, 37 (74.00\%) were discharged whereas $13(26.00 \%)$ patients expired. The majority of patients, irrespective of outcome had Vitamin D deficiency. Although normal Vitamin D levels were observed in relatively higher proportion of patients who were discharged (29 patients) as compared to those who expired (12 patients) yet this difference was not significant statistically $(P=0.481)$ in our study.

In study carried out by Barnett et al., ${ }^{6} 120$ patients of sepsis with acute lung injury were included among these patients 16 (13\%) were found to be Vitamin D sufficient, 32 (27\%) were found Vitamin D insufficient and rest 72 (60\%) were found deficient in Vitamin D. They also had chosen similar cutoff values for deficiency, insufficiency, and sufficiency. There was little difference from Vitamin D levels from our study. This difference can be attributed to various factors including different ethnicity, nutritional status, hospital stay, and sun exposure.

In another study carried out by Azim et al. ${ }^{7} 31$ (19.6\%) patients were found Vitamin D sufficient and rest 127 (80.4\%) were found either Vitamin $D$ insufficient or deficient this prevalence of
Vitamin D deficient is quite consistent with that found in our study.

\section{Conclusion}

Vitamin D deficiency leads to increased risk of mortality in the critically ill patients.

\section{References}

1. M. F. Holick, "Vitamin D: importance in the prevention of cancers, type 1 diabetes, heart disease, and osteoporosis," American Journal of Clinical Nutrition, vol. 79, no. 3, pp. 362-371, 2004.

2. J. S. Adams and M. Hewison, "Update in vitamin D," Journal of Clinical Endocrinology and Metabolism, vol. 95, no. 2, pp. 471-478, 2010.

3. P. Lee, J. A. Eisman, and J. R. Center, "Vitamin D deficiency in critically ill patients," The New England Journal of Medicine, vol. 360, no. 18, pp. 1912-1914, 2009.

4. O. Lucidarme, E. Messai, T. Mazzoni, M. Arcade, and D. Du Cheyron, "Incidence and risk factors of vitamin D deficiency in critically ill patients: results from a prospective observational study," Intensive Care Medicine, vol. 36, no. 9, pp. 16091611, 2010.

5. L. R. Matthews, Y. Ahmed, K. L. Wilson, D. D. Griggs, and O. K. Danner, "Worsening severity of vitamin D deficiency is associated with increased length of stay, surgical intensive care unit cost, and mortality rate in surgical intensive care unit patients," American Journal of Surgery, vol. 204, no. 1, pp. 37-43, 2012.

6. Barnett N, Zhao Z, Koyama T, Janz DR, Wang $C Y$, May AK, et al. Vitamin D deficiency and risk of acute lung injury in severe sepsis and 
Pramod Sood et al, International Journal of Medical and Biomedical Studies (IJMBS)

severe trauma: A case-control study. Ann Intensive Care. 2014;4:5.

7. Azim A, Ahmed A, Yadav S, Baronia AK, Gurjar M, Godbole MM, et al. Prevalence of Vitamin $D$ deficiency in critically ill patients and its influence on outcome: Experience from a tertiary care centre in North India (an observational study) J Intensive Care. 2013; $1: 14$. 